\title{
Prenatal diagnosis of fetal hemivertebra at 20 weeks' gestation with literature review
}

This article was published in the following Dove Press journal:

International Journal of General Medicine

2 July 2010

Number of times this article has been viewed

Michail Varras'

Christodoulos Akrivis ${ }^{2}$

'Obstetrics and Gynecology

Department, "Elena Venizelou"

General Maternity Hospital, Athens;

2Obstetrics and Gynecology

Department, "G Chatzikosta" General

State Hospital, loannina, Greece
Correspondence: Michail Varras

Platonos 33, Politia (Kifisia), I 4563 Athens,

Greece

Tel +302108000604

Email mnvarras@otenet.gr
Abstract: Hemivertebra is a rare congenital spinal malformation, where only one side of the vertebral body develops, resulting in deformation of the spine, such as scoliosis, lordosis, or kyphosis. We present the ultrasonographic features of a fetus with hemivertebra at 20 weeks' gestation confirmed by post mortem radiography and pathological examination. The prenatal literature on this disorder is also reviewed. Useful background information is provided for both clinicians and other health professionals who are not familiar with this condition.

Keywords: hemivertebra, congenital scoliosis, prenatal diagnosis, ultrasonography

\section{Introduction}

Hemivertebra is a rare congenital malformation of the spine, where only one side of the vertebral body develops, resulting in a laterally-based wedge vertebra with half a vertebral body, a single pedicle, and hemilamina. ${ }^{1}$ Other terms used for this lesion are congenital scoliosis, unilateral aplasia of the vertebral body, and complete unilateral failure of formation of the vertebral body. ${ }^{2}$ Hemivertebra can be isolated or associated with defects of other organ systems. We describe our experience of a case of congenital scoliosis due to hemivertebra detected prenatally at 20 weeks' gestation, including the ultrasonographic findings that led to the diagnosis and the post mortem radiological characteristics.

\section{Case report}

The patient was a 26-year-old woman, gravida 1 para 0 , with an unremarkable medical history. The triple test at 16 weeks' gestation was negative, ie, alpha-fetoprotein $42 \mathrm{ng} / \mathrm{mL}$ (multiples of the mean [MoM] = 0.992), estriol $3.8 \mathrm{nmol} / \mathrm{L}(\mathrm{MoM}=1.834)$, free beta- human chorionic gonadotropin $28 \mathrm{ng} / \mathrm{mL}(\mathrm{MoM}=2.806)$. No malformations were detected during ultrasound scanning at the sixteenth week of gestation. At the routine ultrasonographic anatomy scan of the fetus at 20 weeks' gestation, the biparietal diameter was $46 \mathrm{~mm}$, the abdominal circumference $145 \mathrm{~mm}$, the fetal femur length $32 \mathrm{~mm}$, and estimated fetal weight $348 \mathrm{~g}$. Amniotic fluid was within normal limits. However, a distortion at the lumbar region of the spine was demonstrated in the longitudinal plane. A small triangular bony structure was wedged against the normal vertebral bodies (Figures 1 and 2). The overlying skin appeared intact and there were no intercranial findings suggestive of an open neural tube defect. No other abnormalities were detected. The initial diagnosis was single hemivertebra, and the parents opted for termination of pregnancy. Labour was induced using misoprostol. A $500 \mathrm{~g}$ stillborn 


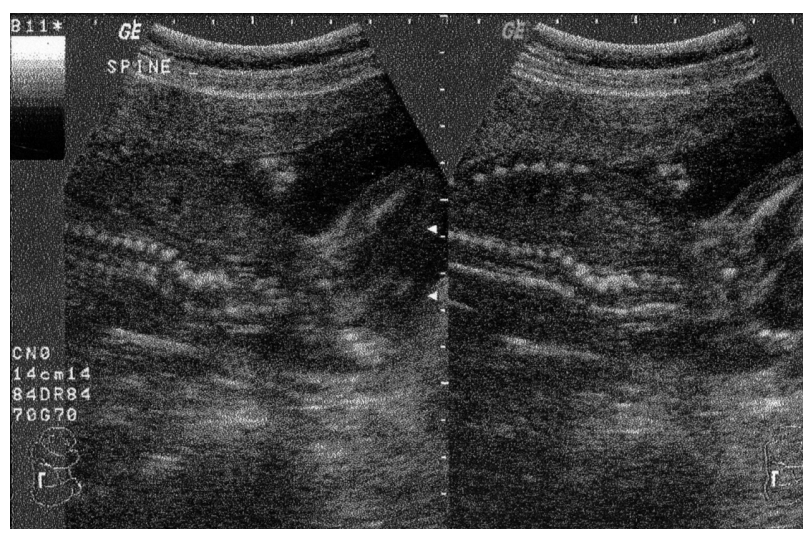

Figure I Longitudinal ultrasonographic scans of the spine of a second trimester fetus with hemivertebra.

female neonate was delivered with obvious deformation of the spine (Figure 3), and the ultrasonographic findings were confirmed with a simple X-ray of the stillborn fetus (Figures 4 and 5) and with pathologic examination. No other skeletal anomalies of the spine, ribs, and limbs, or cardiac, renal, and gastrointestinal anomalies were found. The fetal karyotype was $46, \mathrm{XX}$.

\section{Discussion}

The incidence of hemivertebra is estimated at $0.5-1.0$ per 1000 births, with a male to female ratio of 0.31 for multiple vertebral anomalies and 0.68 for solitary vertebral anomalies. ${ }^{1,3}$ In our case, the affected fetus was female, and this is in agreement with the described higher incidence for females compared with males. The English literature contains only few cases of vertebral malformation which were diagnosed prenatally. ${ }^{2,4-10}$

Gastrulation occuring in the embryonic period converts the bilaminar embryonic disc to a trilaminar structure

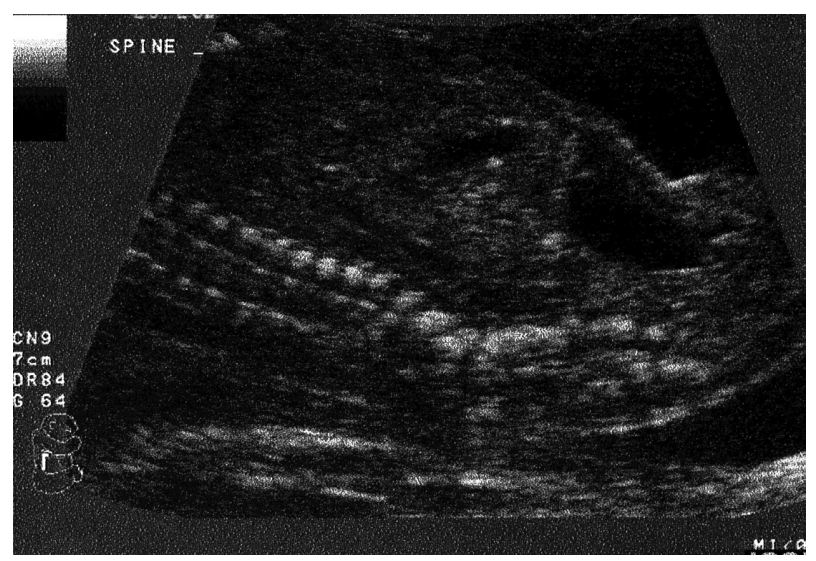

Figure 2 Longitudinal ultrasonographic scan of the spine of a second trimester fetus with hemivertebra.

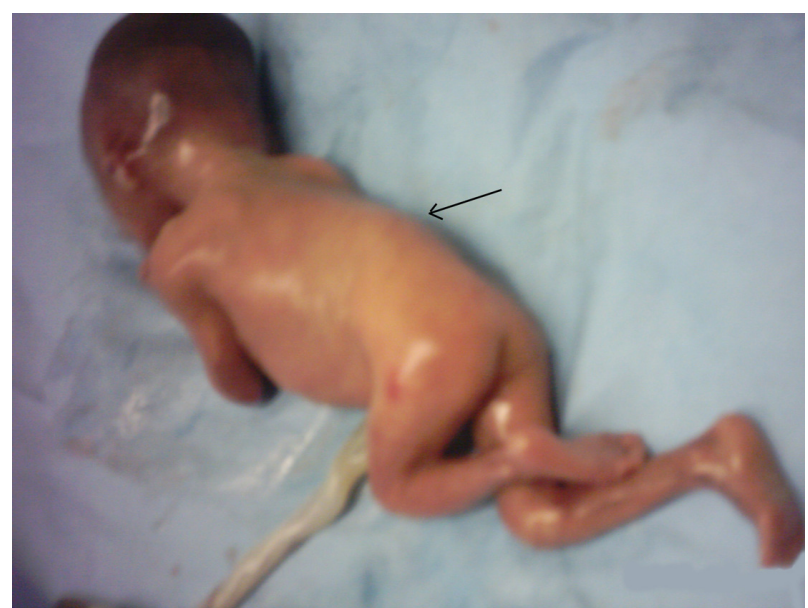

Figure 3 The stillborn female neonate presents obvious deformation of the spine (arrow).

containing ecto-, endo-, and mesoderm. Spinal development involving the formation of mesodermal-derived somites culminates in the formation of primitive vertebrae. ${ }^{11}$ The vertebrae develop during the sixth week of gestation, when two lateral chondrification centers arise in the developing vertebral bodies. These chondrification centers then fuse by seven to eight weeks' gestational age to form the primary ossification center of the vertebral body, transiently separated

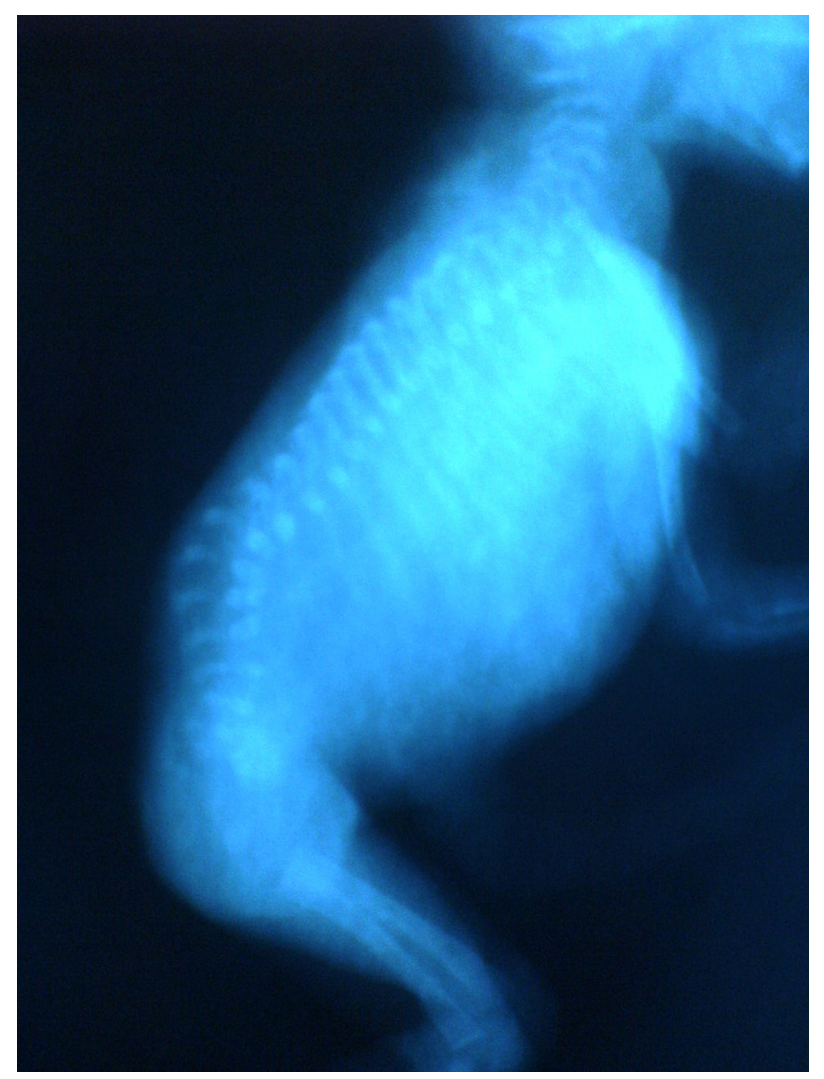

Figure 4 Post mortem lateral radiograph of the spine shows lumbar hemivertebra. 


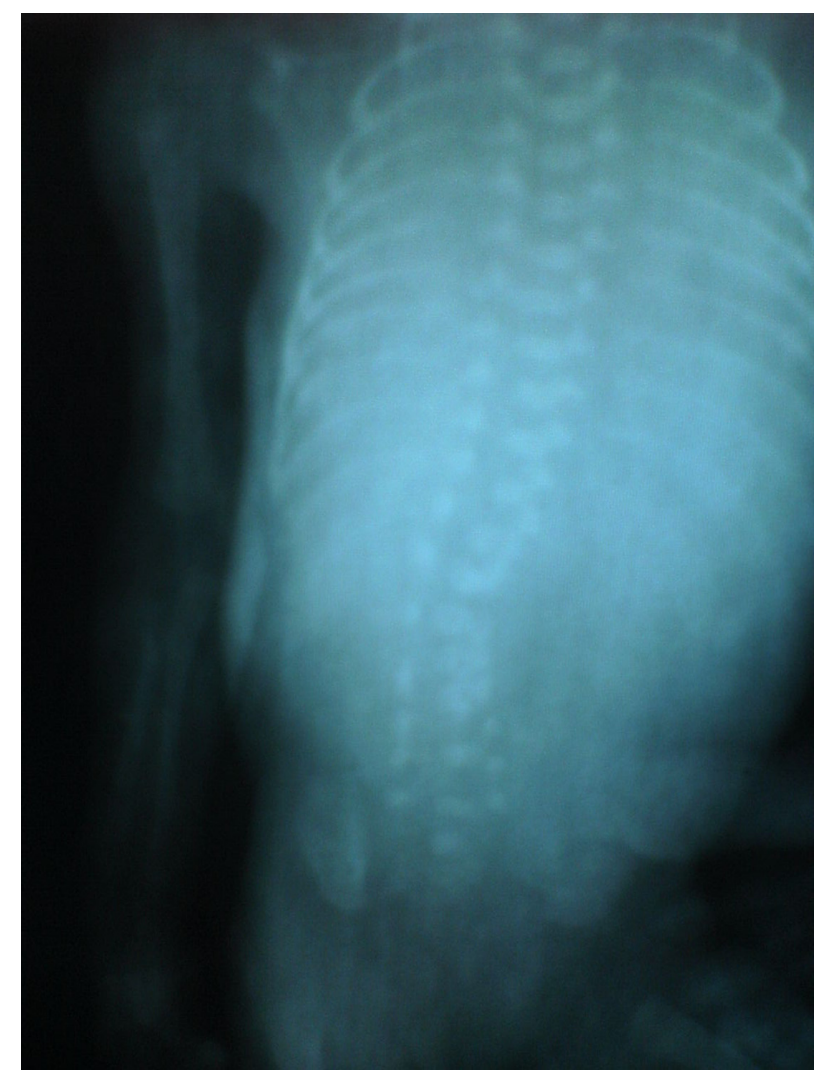

Figure 5 Post mortem plain radiograph of the spine shows lumbar hemivertebra.

into anterior and posterior aspects by the notochord remnant. Lateral hemivertebrae result from lack of development of one of the paired chondral centers. Less commonly, posterior hemivertebrae result from failed anterior ossification..$^{10,12,13}$ The defective vertebra acts as a triangular wedge-shaped ossified structure within the vertebral column, causing contralateral spine deviation at the level of the abnormal vertebra., ${ }^{2,8,10,14}$ There are four different types of hemivertebrae characterized by the presence or absence of a normal disk space adjacent above and below the affected segment, ie, fully segmented (the most common), semisegmented, nonsegmented, and incarcerated (the least common). ${ }^{1}$

The cause of hemivertebra is unknown. One hypothesis has suggested that hemivertebra may result from abnormal distribution of the intersegmental arteries of the vertebral column. ${ }^{15,16}$ The distribution pattern of the anomaly does not implicate a specific environmental or genetic factor. Hemivertebrae may be isolated or may occur in multiple areas within the spine and is frequently associated with other congenital anomalies. ${ }^{9,17,18}$ Hemivertebra is commonly associated with other skeletal anomalies of the spine, ribs, and limbs. ${ }^{9,10,17,18}$ Combination of diastematomyelia and hemivertebra has also been observed. ${ }^{19,20}$ Cardiac and genitourinary tract anomalies are the most common extramusculoskeletal anomalies seen with hemivertebra. Anomalies of the central nervous system and gastrointestinal tract have also been reported. 9,10,17,18 Approximately one-sixth of patients with vertebral body anomalies have associated anomalies unrelated to the axial skeleton and spinal cord. ${ }^{1,3}$ Hemivertebra may be part of syndromes including Jarcho-Levin syndrome, Klippel-Fiel syndrome, VATER syndrome (vertebral anomalies, imperforate anus, tracheoesophageal fistula, and renal anomalies), VACTERL syndrome (VATER with cardiac and limb anomalies), OEIS (omphalocele, bladder extrophy, imperforate anus, and spine anomalies), the Potter sequence, and open spina bifida. ${ }^{13}$ The incidence of karyotypic abnormalities in fetuses with isolated vertebral anomalies is thought to be small.,10

The gestational age at diagnosis of hemivertebra is usually 20-28 weeks. ${ }^{1}$ However, because the ossification centers of the vertebrae can be initially visualized by ultrasound examination after 12 weeks' gestation, the defect of ossification can be visible on early scans. ${ }^{1}$ The diagnosis of fetal hemivertebra on prenatal ultrasound requires meticulous examinations. The sonographic findings associated with fetal hemivertebra include a distortion in the shape of the spine that can be assessed by both sagittal and coronal scans. The deformity manifests as a triangular bony structure, smaller than a vertebra, that acts as a wedge against the normal vertebral bodies (Figure 1). ${ }^{8,10} \mathrm{~A}$ careful assessment for associated cardiac, renal, and gastrointestinal anomalies needs to be performed. Hemivertebra may have a similar ultrasonic appearance to other vertebral abnormalities (wedge vertebra, butterfly vertebra, bloc vertebra, bar vertebra, or any combination of these) that cause congenital scoliosis, open neural tube defects, and diastematomyelia., ${ }^{2,821}$ Diastematomyelia is a rare form of spinal dysraphism, characterized by a sagittal cleft in the spinal cord, conus medullaris, and/or filum terminale with splaying of the posterior vertebral elements. ${ }^{22-24}$ Diastematomyelia is subdivided into Type I and Type II. Type I consists of two hemicords, contained within two separate dural sheaths, and separated by a rigid, osseocartilaginous median septum. ${ }^{22-24}$ Type II is characterized by two hemicords housed in a single dural sheath and separated by a nonrigid, fibrous median septum..$^{22-24}$ Diastematomyelia may be isolated, or it may be associated with other spinal anomalies such as spina bifida, kyphoscoliosis, butterfly vertebra, or hemivertebra. ${ }^{22}$ Diastematomyelia usually occurs as a nonsyndromal sporadic disorder. However, a few familial cases have been reported and Bacli et al suggested that X-linked dominant inheritance, with limitations to females because of lethality in homozygous males, could not be excluded. ${ }^{25}$ 
Ultrasonographic features of diastematomyelia include widening of the spinal canal in the coronal section, with extraechogenic foci located in the midline between localized widenings of the posterior ossification centers of the vertebral column. ${ }^{24}$ Echogenic foci in the posterior aspect of the vertebral column in axial section are highly specific for diastematomyelia. ${ }^{22}$

Obstetric counseling and management for fetal hemivertebra depends on the time of diagnosis, the presence of isolated hemivertebra or of multiple vertebral abnormalities, and the identification of other associated malformations. The option of pregnancy termination should be offered when involvement of several vertebrae or of additional organ systems is observed. If only one vertebra is affected and no other fetal malformations are detected, conservative management of pregnancy could be offered. ${ }^{10}$ In our case, pregnancy termination was performed at the parents' request. The prognosis of hemivertebra is partially determined by the site of the affected vertebra, the number of affected vertebrae, and the associated anomalies. ${ }^{1}$ The prognosis of isolated hemivertebra is good. Left untreated, $25 \%$ of patients with congenital scoliosis show no progression, 50\% progress slowly, and $25 \%$ progress rapidly during growth. ${ }^{2,26}$ When the prenatal diagnosis of hemivertebrae is made, a meticulous search for associated anomalies should be performed. Chromosomal analysis can be offered, especially in the presence of associated anomalies because an increased risk of aneuploidy may exist. Alpha-fetoprotein concentration in amniotic fluid can be assessed if there is a question of an open neural tube defect and the fetus is of appropriate gestational age. Serial ultrasonic evaluation is recommended to follow fetal growth and evaluate for signs of an open neural tube defect that may not be present at an initial early ultrasound. ${ }^{2,8}$ Fetuses with hemivertebra have high rates of cesarean delivery and growth restriction. Neonates with nonisolated hemivertebrae are more often delivered before term and have higher mortality rates. ${ }^{13}$ If there are no other complicating factors, standard management of labor and delivery is recommended. A careful neonatal assessment for associated cardiac and genitourinary anomalies needs to be performed. ${ }^{2,8}$ The surgical treatment of congenital scoliosis is aimed at producing a straight and balanced spine at skeletal maturity. There are three key factors in achieving an optimal spine at maturity, ie, early diagnosis, anticipation of scoliosis, and prevention of deterioration. ${ }^{1}$ Risk of neural tube defects in siblings is increased. ${ }^{8}$ Therefore, it would be reasonable to offer genetic counseling and prenatal diagnosis for neural tube defects to those patients with a previous child with vertebral anomalies. ${ }^{2}$

\section{Conclusion}

Prenatal diagnosis of hemivertebra is feasible. The characteristic ultrasonographic findings in the fetus allow the early detection of hemivertebra, providing parents with valuable information in order to make their decision regarding the fate of the pregnancy. When the parents opt to continue with the pregnancy, early postnatal evaluation and frequent follow-up will optimize treatment and reduce the risk of serious scoliosis and its complications.

\section{Disclosure}

The authors report no conflict of interest in this research.

\section{References}

1. Weisz B, Achiron R, Schindler A, Eisenberg VH, Lipitz S, Zalel Y. Prenatal sonographic diagnosis of hemivertebra. $J$ Ultrasound Med. 2004;23:853-857.

2. Gauthier DW, Meyer WJ. Hemivertebra. The-Fetus.net. Central nervous system. 1993;1-6. Available from: http://www.thefetus.net. Accessed on May 14, 2010.

3. Wynne-Davies R. Congenital vertebral anomalies: Aetiology and relationship to spina bifida cystica. J Med Genet. 1975;12:280-288.

4. Zelop CM, Pretorius DH, Benacerraf BR. Fetal hemivertebrae: Associated anomalies, significance, and outcome. Obstet Gynecol. 1993;81:412-416.

5. Benacerraf BR, Greene MF, Barss VA. Prenatal sonographic diagnosis of congenital hemivertebra. J Ultrasound Med. 1986;5:257-259.

6. Broekman BA, Dorr JP. Congenital kyphosis due to absence of two lumbar vertebral bodies. J Clin Ultrasound. 1991;19:303-305.

7. Henry RJ, Norton S. Prenatal ultrasound diagnosis of fetal scoliosis with termination of the pregnancy: Case report. Prenat Diagn. 1987; 7:663-666.

8. Pilu JL. Hemivertebra. The fetus.net. 1999;13. Available at: (http:// www.thefetus.net). Accessed on May 14, 2010.

9. McMaster MJ, David CV: Hemivertebra as a cause of scoliosis. J Bone Joint Surg. 1986;68:588-595.

10. Goldstein I, Makhoul IR, Weissmaan A, Drugan A. Hemivertebra: Prenatal diagnosis, incidence and characteristics. Fetal Diagn Ther. 2005;20:121-126.

11. Leung YL, Buxton N. Combined diastematomyelia and hemivertebra. A review of the management at a single centre. J Bone Joint Surg. 2005;87B:1380-1384.

12. Moore KL, PeraudTVN. The Developing Human. 5th ed. Philadelphia, PA: W.B. Saunders; 1993.

13. Wax JR, Watson WJ, Miller RC, et al. Prenatal sonographic diagnosis of hemivertebrae - associations and outcomes. JUltrasound Med. 2008; 27:1023-1027.

14. Hefti F. Congenital anomalies of the spine. Orthopade. 2002;31: $34-43$.

15. Tanaka T, Uhthoff HK. The pathogenesis of congenital vertebral malformations. A study based on observations made in 11 human embryos and fetuses. Acta Orthop Scand. 1981;52:413-425.

16. Forrester MB, Merz RD. Descriptive epidemiology of hemivertebrae, Hawaii, 1986-2002. Congenit Anom. 2006;46172-46176.

17. Connor JM, Conner AN, Connor RAC, Tolmie JL, Yeung B, Goudie D. Genetic aspects of early childhood scoliosis. Am J Med Genet. 1987;27: 419-424.

18. David CV: Hemivertebra as a cause of scoliosis. $J$ Bone Joint Surg. 1986;68:588-595.

19. Leug YL, Buxton N. Combined diastematomyelia and hemivertebra. A review of the management at a single centre. J Bone Joint Surg. 2005; 87-B:1380-1384. 
20. Dabra A, Gupta R, Sidhu R, Kochhar S, Kaur L, Singh J. Sonographic diagnosis of diastematomyelia in utero: A case report and literature review. Austral Radiol. 2001;45:222-224.

21. Chen M, Chan B, Lam TP, Shek T, Lee CP, Tang MH. Sonographic features of hemivertebra at 13 weeks' gestation. J Obstet Gynecol Res. 2007;33:74-77.

22. Has R, Yuksel A, Buyukkurt S, Kalelioglu I, Tatli B. Prenatal diagnosis of diastematomyelia: Presentation of eight cases and review of the literature. Ultrasound Obstet Gynecol. 2007;30:845-849.

23. Karaşahim KE, Gezginç K, Alanbay I, Ulubay M, Başer I. Ultrasonographic diagnosis of diastematomyelia during the 14th week of gestation. Taiwan J Obstet Gynecol. 2009;48:163-166.
24. Allen LM, Silverman RK. Prenatal ultrasound evaluation of fetal diastematomyelia: Two cases of type I split cord malformation. Ultrasound Obstet Gynecol. 2000;15:78-82.

25. Balci S, Caglar K, Eryilmaz M. Diastematomyelia in two sisters. Am J Med Genet. 1999;86:180-182.

26. Winter RB. Congenital scoliosis. Orthop Clin North Am. 1988;19: $395-408$.
International Journal of General Medicine

\section{Publish your work in this journal}

The International Journal of General Medicine is an international, peer-reviewed open-access journal that focuses on general and internal medicine, pathogenesis, epidemiology, diagnosis, monitoring and treatment protocols. The journal is characterized by the rapid reporting of reviews, original research and clinical studies across all disease areas.
Dovepress

A key focus is the elucidation of disease processes and management protocols resulting in improved outcomes for the patient.The manuscript management system is completely online and includes a very quick and fair peer-review system. Visit http://www.dovepress.com/ testimonials.php to read real quotes from published authors.

Submit your manuscript here: http://www.dovepress.com/international-journal-of-general-medicine-journal 